\title{
Radial Field Piezoelectric Diaphragms
}

R G Bryant ${ }^{1}$, R T Effinger IV ${ }^{2}$ and B M Copeland $\mathrm{Jr}^{3}{ }^{3}$

NASA Langley Research Center, MS $226^{1}$, MS $390^{3}$, Hampton, VA 23681, USA

${ }^{2}$ NASA-USRP Student, Texas A\&M, Dept of Mech. Eng., College Station, TX 77840, USA

\begin{abstract}
:
A series of active piezoelectric diaphragms were fabricated and patterned with several geometrically defined Inter-Circulating Electrodes "ICE" and Interdigitated Ring Electrodes "IRE". When a voltage potential is applied to the electrodes, the result is a radially distributed electric field that mechanically strains the piezoceramic along the Z-axis (perpendicular to the applied electric field). Unlike other piezoelectric bender actuators, these Radial Field Diaphragms (RFDs) strain concentrically yet afford high displacements (several times that of the equivalent Unimorph) while maintaining a constant circumference. One of the more intriguing aspects is that the radial strain field reverses itself along the radius of the RFD while the tangential strain remains relatively constant. The result is a Z-deflection that has a conical profile. This paper covers the fabrication and characterization of the $5 \mathrm{~cm}$. (2 in.) diaphragms as a function of poling field strength, ceramic thickness, electrode type and line spacing, as well as the surface topography, the resulting strain field and displacement as a function of applied voltage at low frequencies. The unique features of these RFDs include the ability to be clamped about their perimeter with little or no change in displacement, the environmentally insulated packaging, and a highly repeatable fabrication process that uses commodity materials.
\end{abstract}

\section{Introduction}

Piezoelectric materials are widely used as components in electrical and mechanical devices as both actuators and sensors [1]. During the past decade, several novel concepts have emerged in the methodology of fabricating and packaging these piezoelectric ceramics. These innovations have enhanced the displacement, strain energy and durability without changing the intrinsic properties of the active ceramic material. Researchers at NASA Langley have developed several of these devices, and they include the Thunder actuator and the Macrofiber Composite [2-4].

Recently, work has focused on a new actuator, the Radial Field Diaphragm or "RFD" [5]. This actuator/sensor is a packaged piezoelectric ceramic that uses a radially induced electric field to afford conical displacement perpendicular to the applied electric field. There are several ways to induce a radial field using certain types of electrode patterns. Two such designs include Inter-Circulating Electrodes "ICE" and Interdigitated Ring Electrodes "IRE". An additional benefit the radial field provides is that the displacement also works for polygons and thus may not be constrained by geometry. This paper will discuss the effect of these radial electric fields using the ICE and IRE patterns on circular disks and some of the applications for these radial field diaphragms.

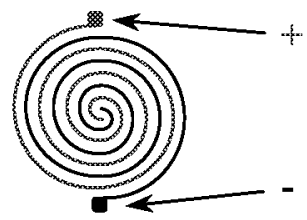

ICE

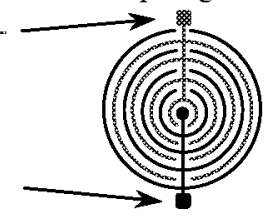

IRE

\section{Experimental}

\section{A. Materials:}

The unelectroded PZT ceramics were obtained from Morgan Matroc Inc. and CTS Wireless Inc. The epoxy adhesive used was E-120HP from Loctite Corp. The polyimide/copper clad used was Pyrolux LF 8051 from Dupont Inc and the release film and press pads were obtained from Pacothane Technologies Inc.

B. RFD Actuator Fabrication Procedure:

a) Assembly of Components

1. All materials were cleaned by wiping with ethanol using a lent-free cloth and handled with latex gloves to prevent contamination.

2. Adhesive was applied across both top and bottom electrode patterns of the copper clad film..

3. A thin coat of adhesive was spread uniformly on one side of the ceramic wafer. The wafer was centered on the bottom electrode pattern, and a thin layer of adhesive was spreadonto the exposed side of the ceramic wafer.

4. The top and bottom electrode patterns were centered against the wafer. Trapped air pockets were removed by carefully applying pressure to the top electrode pattern. The laminate was flipped and the procedure was repeated.

b) Curing Process:

1. A metal plate was placed on a level surface. Two press pads were stacked on top of the plate followed by one sheet of release film and the assembled actuator. 
2. Another layer of release film on top of the actuator followed by two more press pads and a metal plate.

3. The assembly was placed in a vacuum press and evacuated for about 1 minute. The platens were closed to remove any air pockets from between the wafer, epoxy, and electrode patterns. The platens were reopened under vacuum for 10 minutes.

4. The press was heated to $120^{\circ} \mathrm{C}$ and $\sim 700$ MPa pressure was applied for 2 hours.

5. The press was cooled with the pressure and vacuum released prior to removal of the actuator.

C. Characterization:

a) The topographical profile data as a function of DC voltage was acquired using a Brown \& Sharp Chameleon 153010 5S2 Validator; the low frequency displacement data was acquired with the use of a Newport $850 \mathrm{~F}$ LVDT. In both cases a Trek Model 10/10B amplifier was the high voltage supply and the RFD actuators were mechanically constrained $2-3 \mathrm{~mm}\left(\sim 3 / 32^{\prime \prime}\right)$ from the ceramic perimeter using PMMA circular window clamps.

b) Data acquired include out-of-plane displacement data and in-plane strain data. The displacement data was acquired with the use of a Newport 850F LVDT, a Trek Model 10/10B high voltage amplifier, and a Lab-View data acquisition software program. In-plane strain data were acquired with wire-resistance strain gauges from Measurements Group, Inc., a Trek Model 609E-6 high voltage amplifier, and a LabView data acquisition software program. All RFD's were measured constrained about their perimeter.

D. Conversion factors:

$1 \mathrm{mil} \cong 25 \mu \mathrm{m}, 40 \mathrm{mils} \cong 1 \mathrm{~mm}$

\section{Results and Discussion}

A. Background:

When the fabrication of the RFD is complete, two designated sections of dielectric film are removed to expose the copper electrode pads. Wires are then soldered to the pads and the resistance of the wafer is tested for a short circuit. If there are no electrical shorts, the wafer is poled under a DC voltage according to the electrode pattern spacing (Table 1). Once the RFDs are poled, their physical profile changes from flat to conical.

To optimize the design of an actuator, it is important to understand how the aspects of the design, fabrication and test methodology affect and determine the actuator performance. Common methods used to measure the performance of piezoelectric actuators include; strain per volt, strain as a function of frequency, life cycle performance, electro-mechanical efficiency, physical displacement and environmental durability. .Since the RFD's advantage over other piezoelectric devices is its ability to concentrically move out of plane, we wish to optimize the RFD's vertical displacement per volt. The displacement of a RFD is defined as the Z-axis movement beyond its poled position in response to an applied voltage. Lastly, performing all measurements on the RFD's as mounted assures better correlation between experimentally determined results and real world applications.

B. Nomenclature:

A descriptor has been adopted to help distinguish between the different electrode configurations using both the ICE and IRE architectures. As shown in Figure 1, two numbers separated by a " $/$ " will be used to describe the electrode patterns. The first number is the electrode line width and the second number is the center-to-center spacing between electrodes, all in mils. The other necessary physical distinctions between RFD designs, namely, the ceramic thickness, dimensions and composition are indicated
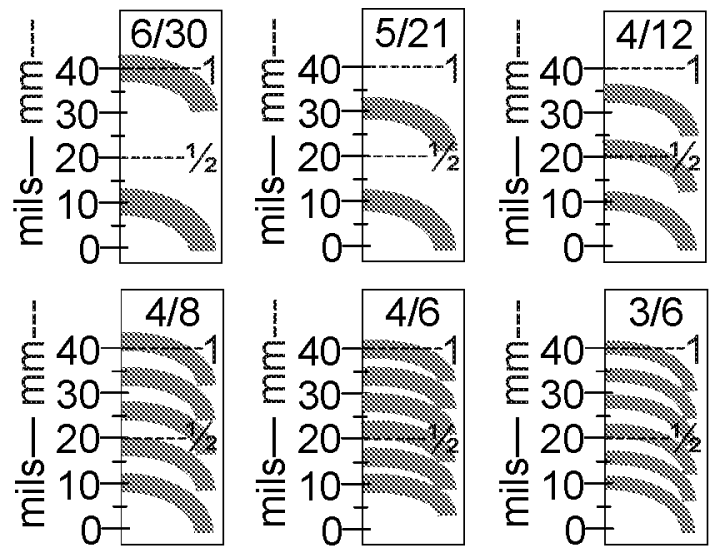

Figure 1. Electrode Pattern Designations.

There are several factors that determine the RFD's performance. These include:

a) Ceramic Composition and Geometry - imparts the base electrical and mechanical behavior;

b) Line Spacing/Pattern - determines the electric field density and vectors;

c) Applied Voltage, Frequency and Strain effects displacement; and

d) Adhesive and Dielectric Package - bestows operational temperature range, processing parameters, dielectric behavior and ultimate durability. 
This paper examines factors a), b), and c) at low frequency under no load conditions. In order to do this, several 2" diameter RFDs were made of each line pattern and poled under the conditions determined from the hysteresis butterfly curves (Table 1).

C. Electrical Characterization:

Table 1

DC Poling and Operational Voltages Ranges.

\begin{tabular}{|c|c|c|c|}
\hline $\begin{array}{c}\text { Line } \\
\text { Pattern }\end{array}$ & $\begin{array}{c}\text { DC Poling } \\
\text { Voltage }^{*}\end{array}$ & $\begin{array}{c}\text { Positive } \\
\text { Volts -Max }\end{array}$ & $\begin{array}{c}\text { Negative } \\
\text { Volts - Max }\end{array}$ \\
\hline $6 / 30$ & 2500 & 2500 & -1100 \\
\hline $5 / 21$ & 1800 & 1800 & -1000 \\
\hline $4 / 12$ & 1300 & 1300 & -800 \\
\hline $4 / 8$ & 1000 & 1000 & -600 \\
\hline $4 / 6$ & 800 & 800 & -600 \\
\hline
\end{tabular}

*2 min. at room temperature.

The general trend shown on Table 1 is that as the line spacing ratio (center to center distance/line width) decreases, the poling voltage and the maximum voltage levels decline. However, this effect is nonlinear especially at the lower ratios. This effect could be geometric, or a shifting of the electrode patterns on the wafer during thermal processing. The resulting misalignment of these patterns will have a greater effect on the symmetry of the electric field at the lower spacing ratios, as any pattern displacement is constant rather than geometrically dependent. Lastly, no data could be obtained for the $3 / 6$ pattern as this configuration had shorts and dropouts resulting from chemical etching.

\section{Characterization using DC Voltage:}

In order to verify the theory that these RFDs displace concentrically and are not bending actuators, the topological profiles were measured at different voltage levels using a cooridnate axis validator. As shown in Figure 2, the RFD was fully clamped about its perimeter during the scans.

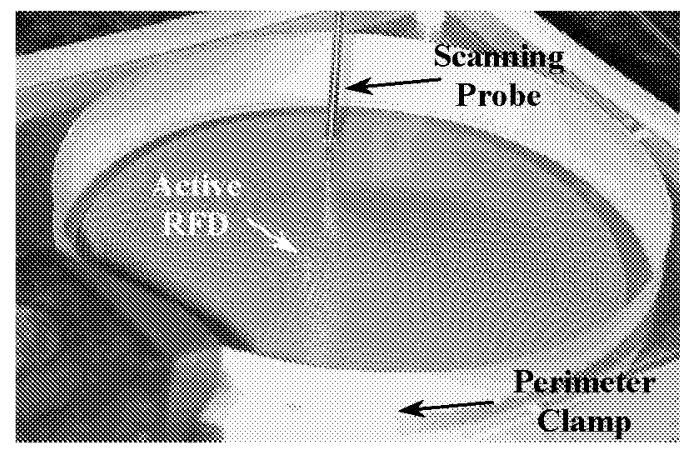

Figure 2.Topological Profile Scanning of a Mounted RFD at Different Voltage Levels.

RFDs ranging in size from $2.5 \mathrm{~cm}$ to $7.5 \mathrm{~cm}(1 "$ to 3") using different line patterns were scanned in this fashion. The results of the $5 \mathrm{~cm} 4 / 12$ patterns for both the ICE and IRE patterns are shown in Figures $3 a$ and $b$ and are representative of all the RFDs scanned.

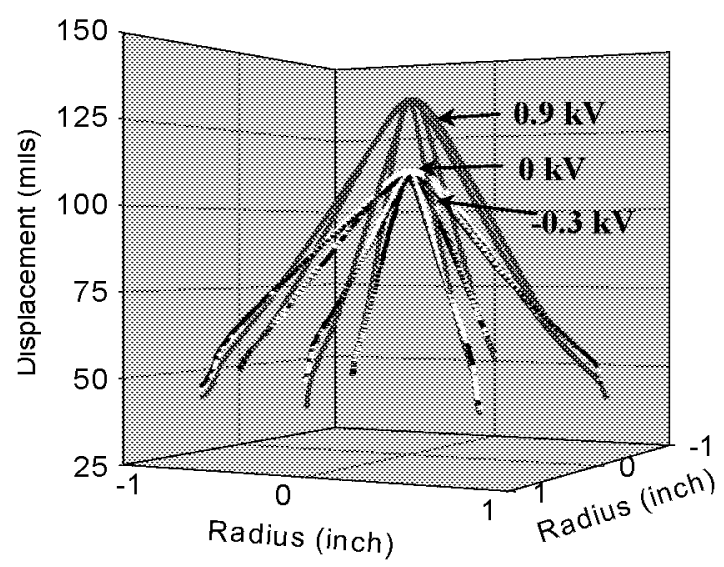

Figure 3a. Topological Profile of $5 \mathrm{~cm}$ RFD with 4/12 ICE Electrode Pattern

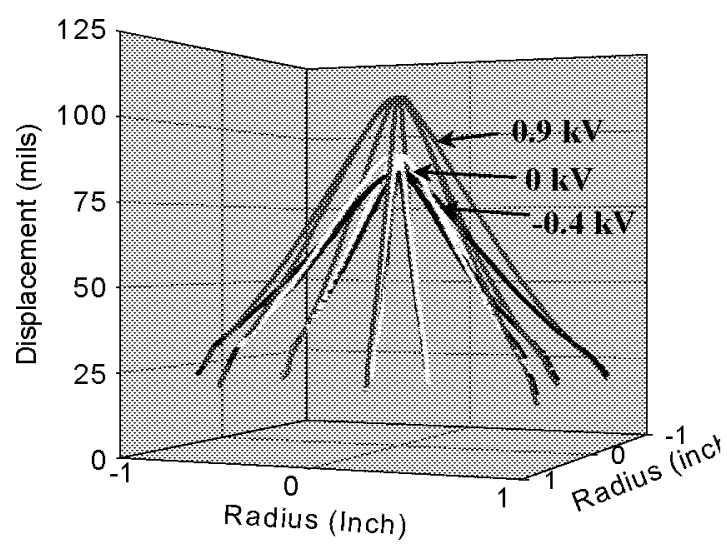

Figure 3b. Topological Profile of $5 \mathrm{~cm} \mathrm{RFD} \mathrm{with}$ 4/12 IRE Electrode Pattern

There are several things to notice in Figure $3 \mathrm{a}$ and $3 \mathrm{~b}$. First, the cross sectional slices of the RFD are triangular (pyramidal) rather than Gaussian or semicircular. Secondly, this view shows concentric deformation at all voltages. Lastly, once poled, these RFD do not sit flat (zero volts), and decrease in height when a voltage (negative) is applied with a polarity opposite to that of the poling field. These results were consistent for all RFD diameters, line patterns, thickness and voltages tested. No asymmetric bending was ever seen.

\section{E. Line Spacing:}

The effect of the ICE and IRE line spacing on the 5 $\mathrm{cm}$ RFD's was also measured at various DC voltage levels. The first comparison of the ICE and IRE line patterns are shown in Figure 4 using the $6 / 30$, $5 / 21$ and $4 / 12$ configurations. The next plot (Figure

Proceedings, Actuator 2002, editor Herman Borgmann, Paper A1.3, June 10-12 (2002). 
5) illustrates the effect of the 5 ICE line patterns, $6 / 30,5 / 21,4 / 12,4 / 8$ and $4 / 6$, have on the deflection of $5 \mathrm{~cm}$ RFDs.

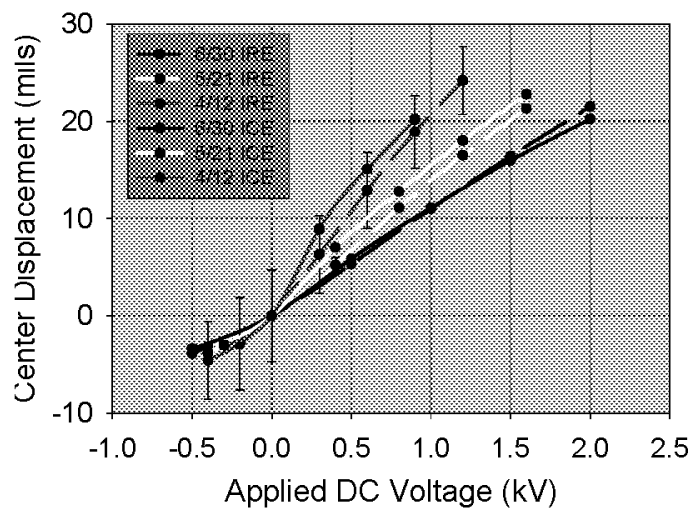

Figure 4. Comparison of the DC Displacement per Volt of ICE and IRE Configurations Using $5 \mathrm{~cm}$ dia. 7 mil Thick 5A-type Ceramic

Figure 4 demonstrates that equal electrode spacing and thickness have the same effect using either pattern type. This results because both patterns introduce the radial field vectors into the ceramic and the electric field intensity as determined by the line pattern. Hence, similar line patterns afford identical field intensity per volt that result in equivalent displacement per volt.

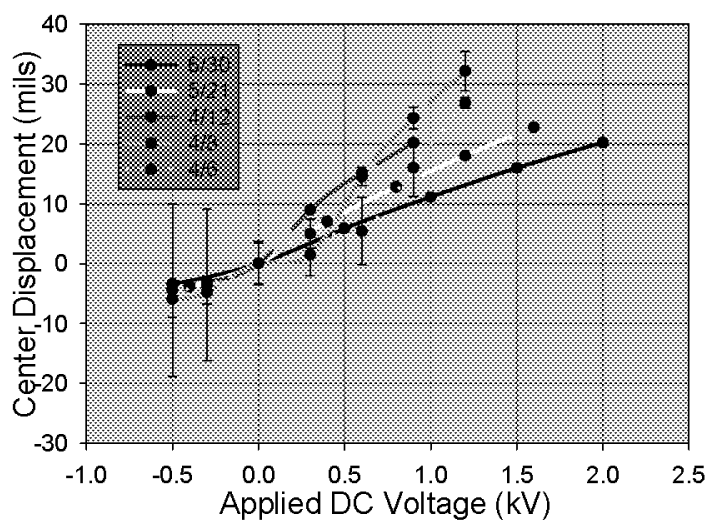

Figure 5. Displacement per Volt of $5 \mathrm{~cm}$ ICE Patterned RFD Using 7 mil Thick 5A-type Ceramic.

As expected, Figure 5 displays the general trend that as the line spacing ratio decreases, the displacement per volt increases (line slope). This effect is expected because the electric field increases per volt as the electrode lines are moved closer together. However, the $4 / 8$ and $4 / 6$ patterns display trends that did not correlate with this observation. This outcome is most likely caused by pattern misalignment, which was reflected in the larger data scatter, rather than a geometrical manifestation.

F. Effect of Ceramic Composition:
As stated earlier, the composition of the piezoelectric ceramic imparts the baseline electrical and mechanical activity to the device of which it is a component. This effect is illustrated in Figure 6 where both ICE and IRE line patterns were compared (see Figure 4) and two PZT ceramic compositions CTS $3195 \mathrm{HD}$ and 3203HD (5A and $5 \mathrm{H}$ type, respectively).

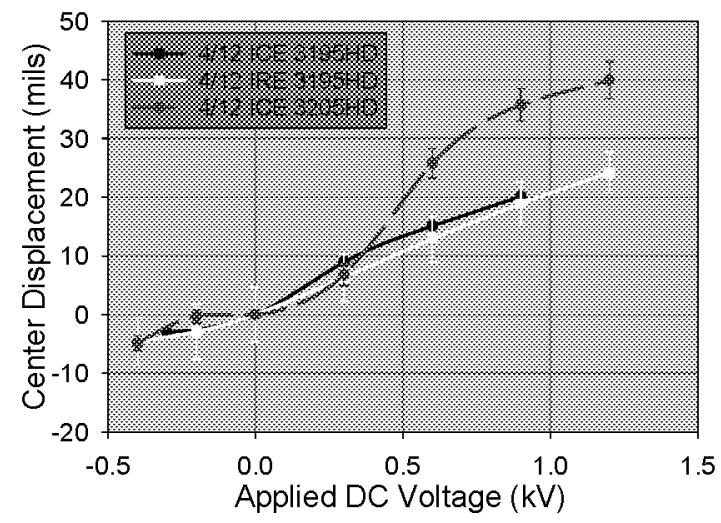

Figure 6. Comparison of $5 \mathrm{~cm}$ dia. 7 mil Thick RFDs at 4/12 Patterns Using Two Ceramic Compositions and Electrode Configuration

As previously shown, similar electrode patterns result in equal activity. Also demonstrated is the role of the ceramic in affecting the RFDs performance. The differences in the CTS 3195 (5A) versus the $3203(5 \mathrm{H})$ include a " $\mathrm{d}$ " and "g" constants that are $\sim 40 \%$ greater for the $5 \mathrm{H}$. However, the trend at lower electric fields does not display an enhancement in performance. The most likely cause of this is due to deviations (electrode misalignment) in the fabrication of these RFDs using the closer line spacing. This may result in lower than expected performance under a moderate electric field

G. Tangential and Radial Strain Behavior:

The localized strain fields of several RFD electrode patterns were measured. All RFD electrode patterns induce a similar strain field into the ceramic, with the only difference being an increase in strain field intensity with decreasing electrode spacing. This effect is expected as decreasing the distance between electrodes increases the electric field strength at a given voltage.

Strain measurements of an unconstrained RFD were taken in the radial and tangential directions, i.e. parallel and perpendicular to the radial electric field. The strain gauge setup and actual test article are shown in Figure 7 where the small "m" patterns are radial and tangential strain gauges. A total of fifteen strain measurements were taken both in the tangential and radial directions with gauges fourteen and fifteen placed beyond the ceramic disk. Because the RFD is circular, it can be assumed that 
the strain field is symmetric about the RFD as evidenced in the topological profile in Figures $3 \mathrm{a}$ and $3 b$. The elliptical pattern for the gauge layout was selected as to allow many gauges without risking wire shorts or spurious electrical/mechanical signals.

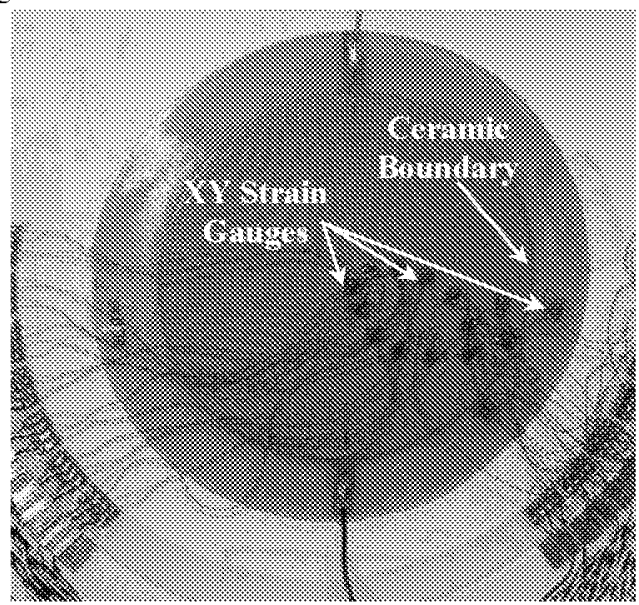

Figure 7. $7.5 \mathrm{~cm}$ RFD Test Specimen.

A $0.1 \mathrm{~Hz}$ sinusoidal voltage signal was applied to the RFD and both the radial and tangential strains were recorded. The strain measurements over four cycles were recorded and averaged. Each strain gauge produced a hysteresis loop commensurate with the nonlinear behavior inherent in piezoelectric ceramics. A linear approximation of each hysteresis loop was calculated and values were translated from the linear fit at voltage levels of $-500,0,500,1000,1500$ and 2000 (est.) volts. This process was repeated for all 30 strain gauge hysteresis loops. These linear strain approximations were then plotted vs. their radial location on the RFD. Repeating this process for the remaining strain gauges creates radial and tangential strain profiles along the radius of the RFD. The radial and tangential strain profiles for $5 / 21$ electrode pattern are presented in Figures 8a and $8 \mathrm{~b}$ respectively.

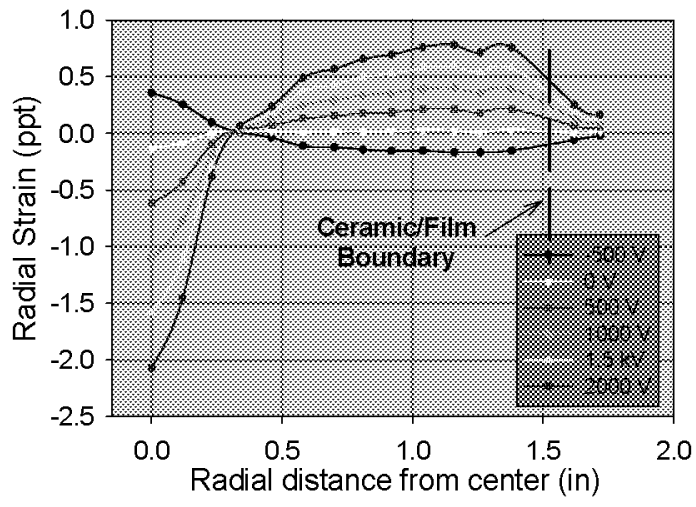

Figure 8a. $7.5 \mathrm{~cm}$ RFD Radial Strain Field 5/21 ICE pattern

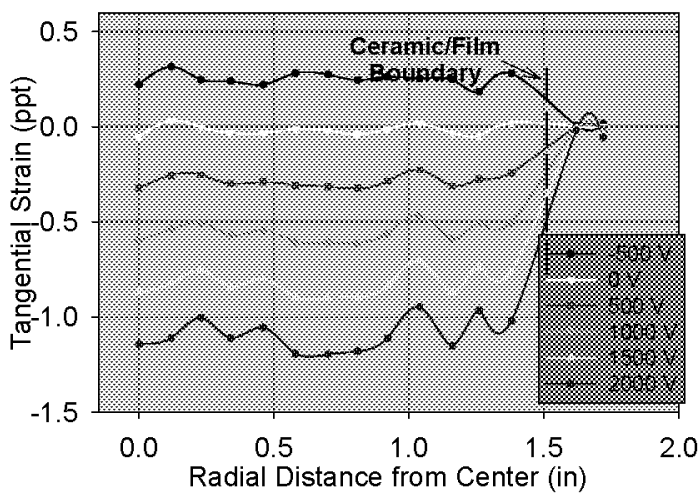

Figure 8b. $7.5 \mathrm{~cm}$ RFD Tangential Strain Field 5/21 ICE pattern.

The above localized radial and tangential strain field results were unexpected. It is important to realize that the trends in the resulting strain fields are reproducible (we used two RFDs with 6/30 and 5/21 ICE patterns), independent of line pattern (except in magnitude) and lend some insight into the unexpected deformation characteristics of the RFDs. The first thing to indicate is that the data points are connected by a spline fit and that these points do not create a smooth contour. This result can be explained as some of these gauges span one or more electrodes that are physical bumps beneath the gauges, resulting in some local alteration of the strain field. Also notice that the strain field drops to almost zero at two places, beyond the ceramic boundary (radial and tangential) and at an inflection point approximately $1 \mathrm{~cm}(\sim 0.25 \mathrm{in})$ from the center. What appears to be occurring is that as a voltage is applied in the same polarity as the poling polarity, the RFD circumference shrinks (negative strain) and the radial strain goes from negative through zero to positive within the ceramic. The effects are reversed when a voltage opposite to the poling polarity is applied. The effect of this and other evidence leads to the conclusion that the global radial strain is approximately zero, the inflection point does not shift, the tangential strains are relatively constant (at the same voltage level) across the radius of the ceramic disk and the strain levels beyond the ceramic are negligible. Thus, the almost straight-edged pyramidal shape of the RFD (Figure $3 a$ and $3 b$ ) may be attributed to the linear tangential strain field, while the minimal strain level beyond the ceramic disk can be attributed to the nearly zero global radial strain and the rapid drop off of the tangential strain. These results lend credibility to the initial observation that the circumference of the ceramic disk remains relatively constant as the RFD actuates, unlike a bending actuator. Lastly, these results also show that the electric field $\mathbf{E}$ (radial) is perpendicular to the global strain field $\boldsymbol{\varepsilon}$ (tangential) while the displacement vector $\mathbf{D}$ is orthogonal to both fields (Figure 9). 


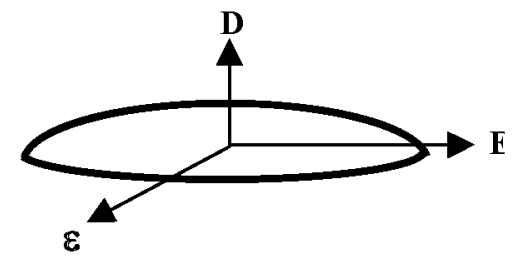

Figure 9. RFD Field Vector Description

H. Devices Using the RFD as an Active Element: Since the RFD displaces concentrically, with negligible strain beyond the perimeter of the ceramic, this active element would lend itself to applications requiring simplistic fixturing about its perimeter to create an easily sealed diaphragm. Such applications include fluidic pumps and valves based on diaphragms. Two basic working pump designs are shown in Figure 10. These schematics demonstrate a single chamber diaphragm pump and a dual chamber diaphragm pump (can use both sides of the diaphragm).
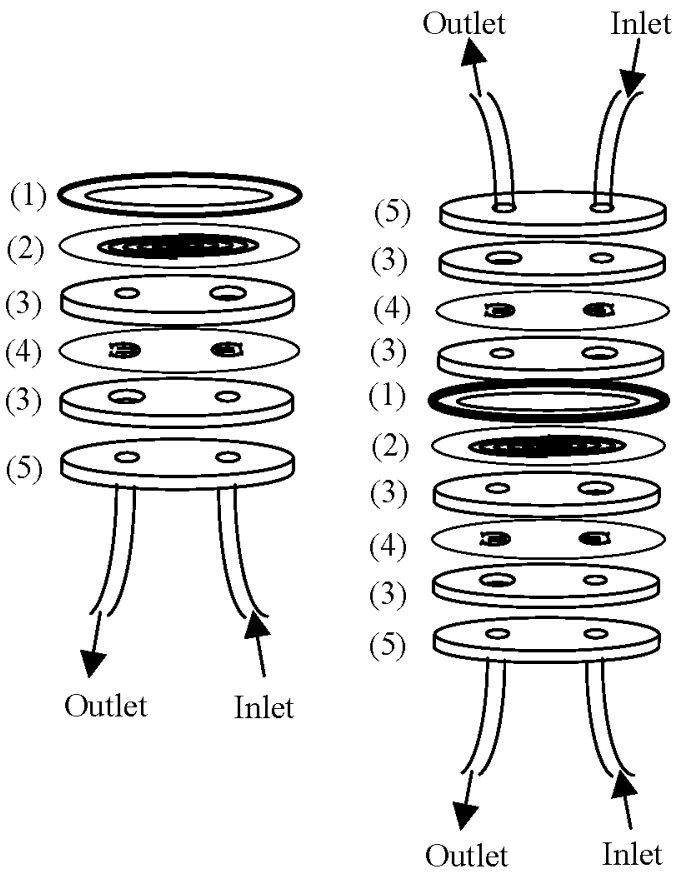

Figure 10. Single and Dual Chamber Diaphragm RFD Pumps.

These pumps comprise a spacer/clamp ring (1), one RFD (2), reversible valve guides (3), a flapper valve assembly (4), and a manifold (5) (see Figure 10). Pumps of this type can be connected in series or parallel to increase fluid flow pressure or volume in a manner analogous to voltage and amperage power schemes used with multiple dry cell batteries. Additionally, these pumps can be staged to operate in a peristaltic fashion where the diaphragm strokes are phased to allow for a diaphragm to either pump fluid or serve as an active valve to allow for pressure buildup and primer-less pumping action.

\section{Conclusions:}

The discovery of the effect of introducing a radial electric field into an electroactive ceramic has introduced a piezoelectric actuator that exhibits unique behavior. This has opened the door to realistic piezoelectric devices such as fluidic pumps, sonic transducers and valves. The method required to fabricate the RFDs is straightforward and uses conventional technology and materials. Ongoing work at NASA will include further characterization at higher frequencies, life cycle testing, force output, refined fabrication procedures, examining different ceramic geometries including polygons and device testing.

\section{References:}

[1] Jaffe, B., Cook, W. R., Jr., and Jaffe, H., Piezoelectric Ceramics, Academic Press Ltd., 1971

[2] K. M. Mossi, G. V. Selby and R. G. Bryant, Thin-Layer Composite Unimorph Ferroelectric Driver and Sensor Properties, Materials Letters, $\underline{35}(1), 39,(1998)$.

[3] Copeland, B. M., Buckley, J. D., Bryant, R. G., Fox, R. L. and Hellbaum, R. F., Ceramic Transactions, 101, 97, (2000).

[4] W. K. Wilkie, R. G. Bryant, J. W. High, R. L. Fox, R. F. Hellbaum, A. Jalink, Jr., B. D. Little, P. H. Mirick, Proceedings of the SPIE Smart Structures and Materials: Industrial and Commercial Appl. Of Smart Structures Tech., $\underline{3991}, 323,(2000)$

[5] R. G. Bryant, R. T. Effinger IV, I. Aranda Jr., B. M. Copeland Jr. and E. W. Covington III, Proceedings of SPIE, Smart Structures and Materials Active Materials : Behavior and Mechanics, Paper 4699-40, San Diego, CA (2002). 